\title{
Successful Endoscopic Resection of Gastric Mucosa-Associated Lymphoid Tissue Lymphoma Unresponsive to Helicobacter pylori Eradication Therapy
}

\author{
Jeongmin Choi \\ Department of Internal Medicine, Sanggye Paik Hospital, Inje University College of Medicine, Seoul, Korea
}

Eradication of Helicobacter pylori is the first-line treatment for gastric mucosa-associated lymphoid tissue (MALT) lymphomas; however, lesions may persist in $20 \%$ of patients after initial treatment, thereby necessitating the use of an additional therapeutic approach. Other treatment options include radiation therapy, chemotherapy, endoscopic resection, rituximab therapy, or watchful waiting. We present a case of localized gastric MALT lymphoma that did not respond to $H$. pylori eradication therapy. The patient waited for 12 months but the tumor showed no signs of regression endoscopically. Histologic examination revealed residual MALT lymphoma. The tumor was then successfully treated using endoscopic submucosal dissection and the patient remained disease-free for 4 years. To our knowledge, this is the first case in which a gastric MALT lymphoma was treated with endoscopic submucosal dissection. In conclusion, endoscopic resection may be recommended as second-line therapy for properly selected patients with gastric MALT lymphoma as it is effective and minimally invasive. Clin Endosc 2022;55:136-140

Key Words: Endoscopic resection; Endoscopic submucosal dissection; Marginal zone B-cell lymphoma; Stomach

\section{INTRODUCTION}

According to the World Health Organization in 2008, mucosa-associated lymphoid tissue (MALT) lymphomas are classified as under marginal zone B cell lymphomas. ${ }^{1}$ In general, most gastric MALT lymphomas are closely linked to chronic Helicobacter pylori infection and about $80 \%$ of low-grade MALT lymphomas regress in response to $H$. pylori eradication therapy. ${ }^{2,3}$

However, some gastric MALT lymphomas are resistant to $H$. pylori eradication therapy and require additional treatment options such as radiotherapy, chemotherapy, endoscopic re-

Received: August 23, 2020 Revised: September 21, 2020

Accepted: September 22, 2020

Correspondence: Jeongmin Choi

Department of Internal Medicine, Sanggye Paik Hospital, Inje University College of Medicine, 1342 Dongil-ro, Nowon-gu, Seoul 01757, Korea

Tel: +82-2-950-1001, Fax: +82-2-950-6701, E-mail: doct00@hanmail.net

ORCID: https://orcid.org/0000-0001-6976-9887

(c) This is an Open Access article distributed under the terms of the Creative Commons Attribution Non-Commercial License (http://creativecommons.org/ licenses/by-nc/3.0) which permits unrestricted non-commercial use, distribution, and reproduction in any medium, provided the original work is properly cited. section, rituximab therapy, or watchful waiting.

We present a case of localized gastric MALT lymphoma that did not respond to initial $H$. pylori eradication therapy, which was successfully treated with endoscopic submucosal dissection (ESD). We recommend treatment options for localized gastric MALT lymphoma.

\section{CASE REPORT}

A 42-year-old woman was referred to our hospital for gastric mass. She had undergone breast-conserving lumpectomy for stage I breast carcinoma 6 months ago.

Upper endoscopy showed an elevated nodular lesion (type IIa) measuring $1.5 \mathrm{~cm}$ in the gastric mid-body (Fig. 1A, B). Biopsy revealed dense small B-lymphocyte infiltration with lymphoepithelial lesions; these findings were consistent with MALT lymphoma. A rapid urease test was performed and revealed a positive result, while histologic examination was positive for $H$. pylori infection. Endoscopic ultrasonography showed a hypoechoic lesion with involvement of the mucosal and submucosal layers; no regional lymph node enlargement 

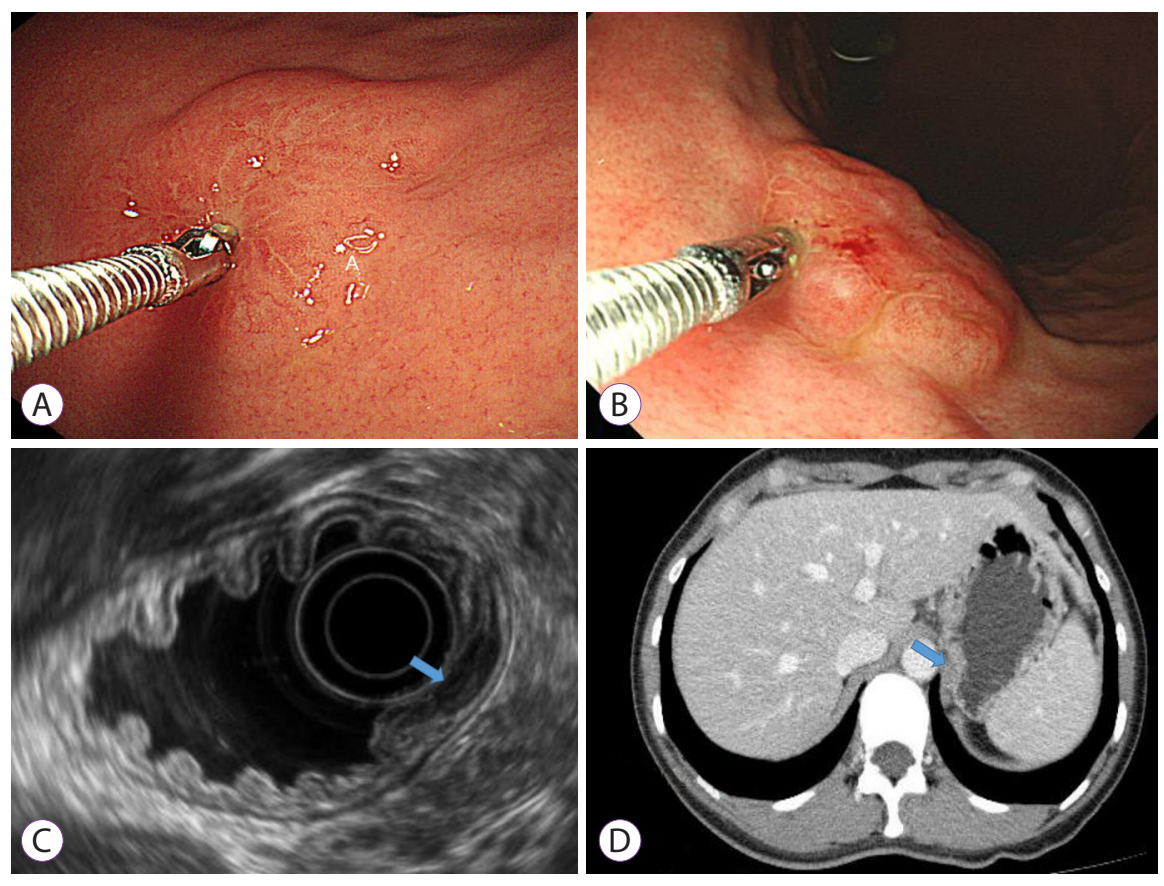

Fig. 1. Gastric mucosa-associated lymphoid tissue lymphoma. (A, B) Upper endoscopy revealed an elevated, nodular mass on the posterior wall of the gastric mid-body. (C) Endoscopic ultrasonography showed a hypoechoic mass invading the mucosal and submucosal layers (arrow). (D) Computed tomography revealed thickening of the gastric wall (arrow).

was found (Fig. 1C). Abdominal computed tomography showed gastric wall thickening in the mid-body and small (less than $1 \mathrm{~cm}$ in size) lymph nodes in the left para-aortic and peri-gastric areas (Fig. 1D). Positron emission tomography did not show abnormal fluorodeoxyglucose uptake. Bone marrow examination showed no bone marrow involvement. Therefore, the patient was diagnosed with a stage IE (Lugano modification of Ann Arbor lymphoma stage $)^{4}$ gastric MALT lymphoma localized to the stomach. She received standard $H$. pylori triple therapy for 14 days which included amoxicillin, clarithromycin, and a proton pump inhibitor (PPI). Three months after completion of $H$. pylori eradication therapy, her urea breath test had a negative result and she was considered cured of H. pylori infection.

Follow-up endoscopy was performed 4 months after $H$. $p y$ lori eradication therapy. The lesion showed no changes endoscopically but the patient's rapid urease test remained negative for H. pylori. Histologically, according to the post-treatment histologic grading system proposed by the Groupe d'Etude des Lymphomes de l'Adulte (GELA), ${ }^{5}$ the patient still had nodular lymphoid infiltrates and several lymphoepithelial lesions, which are suggestive of responding residual disease (rRD).

Follow-up endoscopy was performed 7 months after eradication therapy and showed a protruding mass but with no visible changes. A follow-up endoscopy 12 months after eradication therapy revealed the persistence of a nodular lesion, and pathologic examination revealed lymphoepithelial lesions; these findings were compatible with $\mathrm{rRD}$.

The patient was initially advised to perform careful surveillance since the lesion showed histologic response to $H$. pylori eradication therapy. However, the patient was afraid of the possibility of progression to a malignant lymphoma, since she was a breast cancer survivor, and wanted definitive treatment. After discussing treatment options, which included endoscopic resection, chemotherapy, or radiotherapy, she decided to undergo endoscopic resection.

We resected the gastric MALT lymphoma using ESD. The endoscopist placed marking dots outside the tumor margin using argon plasma coagulation (Fig. 2A). Afterwards, normal saline mixed with indigo carmine was injected into the submucosal layer. After an incision was made, circumferential mucosal incision (Fig. 2B) and submucosal dissection were performed (Fig. 2C). The tumor was resected en bloc (Fig. 2D). There were no complications related to the procedure.

Histologic examination revealed dysmorphic lymphocytic infiltration and several lymphoepithelial lesions (Fig. 3A, B) and immunohistochemical staining was positive for CD-20 and negative for CD-3 (Fig. 3C, D), which were diagnostic for MALT lymphoma. The depth of the tumor was classified as minute submucosal invasion $(<500 \mu \mathrm{m}$ from the muscularis mucosae) with clear lateral and deep resection margins. Six months later, a surveillance endoscopy found a scar but no recurrence. The patient remained disease-free for 4 years. 


\section{Ce cuncan Enosscopy}
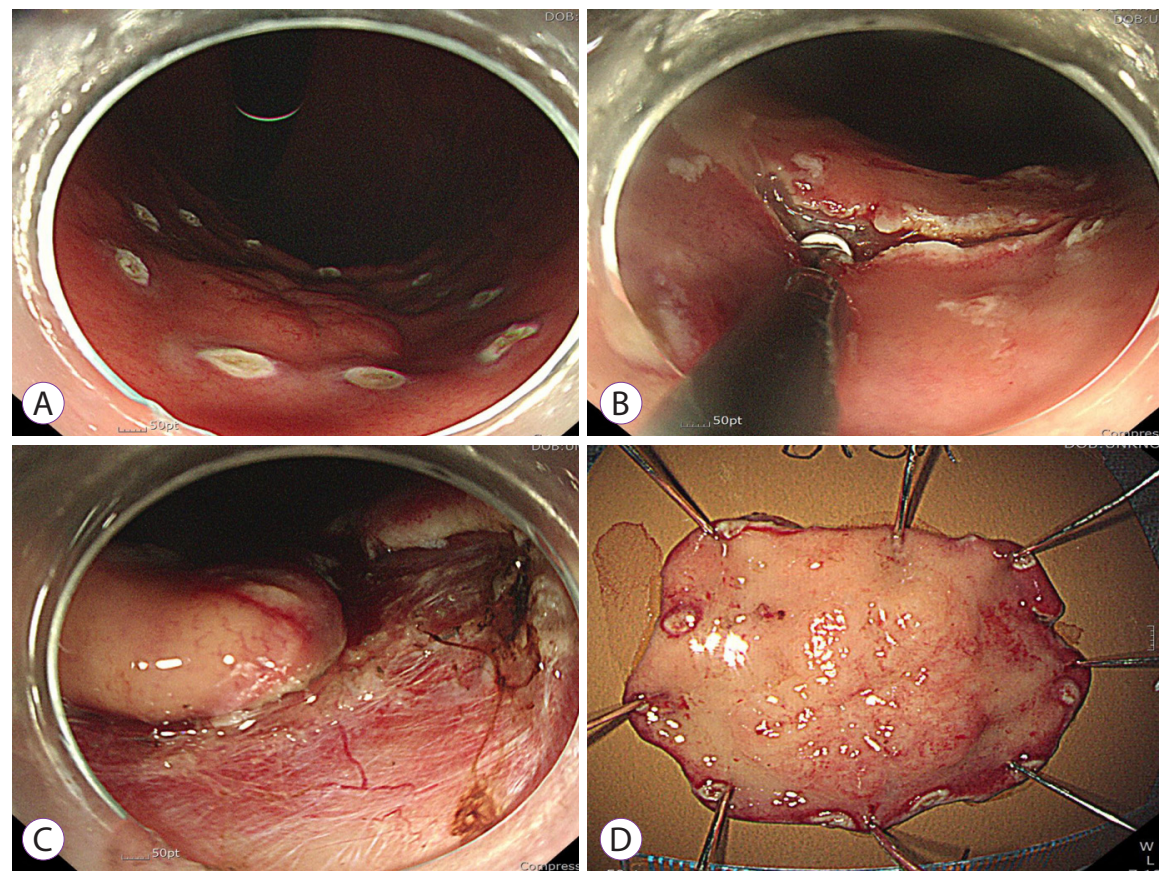

Fig. 2. Endoscopic submucosal dissection procedure. (A) Marking dots outside of the tumor margin. (B) Circumferential mucosal incision. (C) Submucosal dissection. (D) En bloc resection of the tumor.
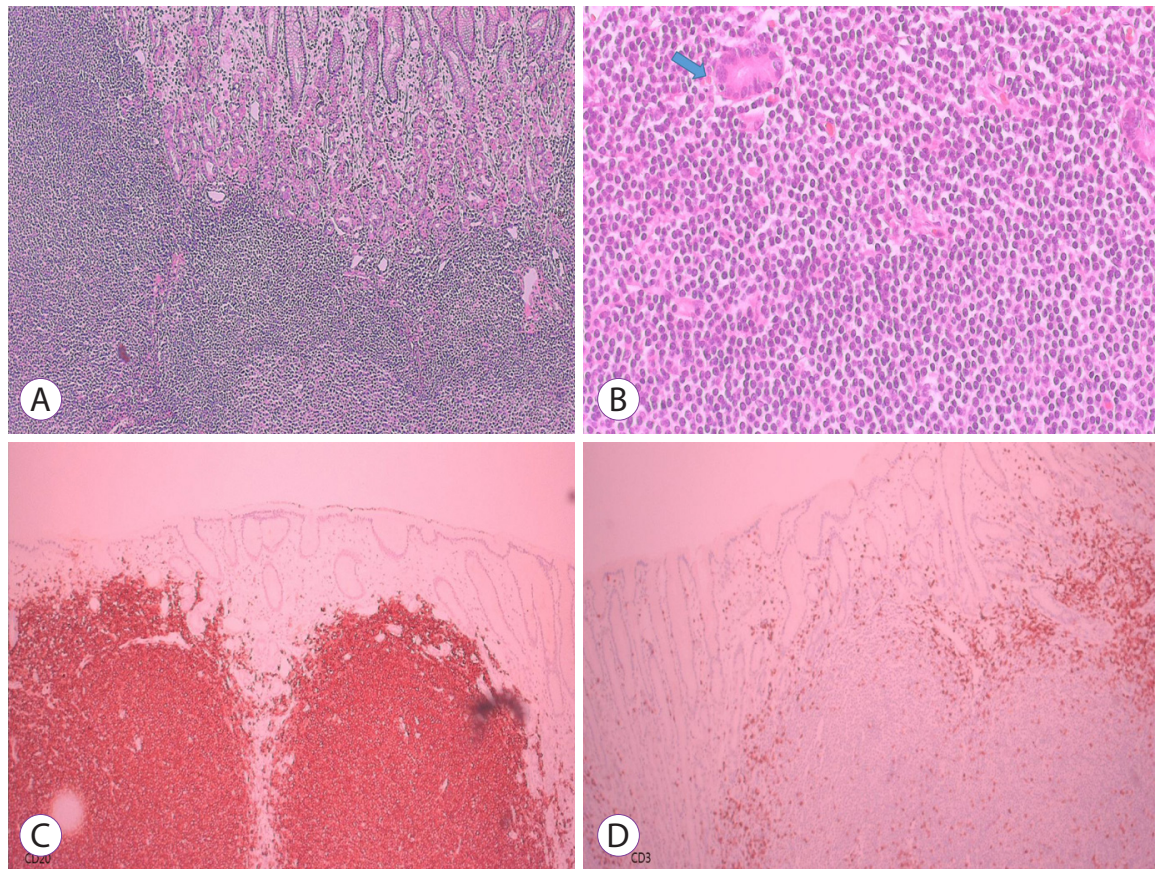

Fig. 3. Histologic findings. (A) Diffuse dense infiltration of small, atypical lymphocytes (hematoxylin and eosin stain, $\times 100)$. (B) Polymorphous lymphoid infiltrates and lymphoepithelial lesions (arrow) (hematoxylin and eosin stain, $\times 400$ ). Immunohistochemical staining was positive for CD-20 (C) but negative for CD-3 (D). 


\section{DISCUSSION}

We initially treated gastric MALT lymphoma with $H$. pylori eradication therapy. However, even when $H$. pylori infection was cured, the lesion did not regress. The patient waited 12 months for the lesion to regress; however, endoscopic findings remained unchanged and histopathologic examination showed residual MALT lymphoma. Ultimately, we treated the patient using endoscopic resection.

Confirmation of eradication should be done in all patients treated for H. pylori. Accurate confirmation is particularly important since approximately $20 \%$ of patients fail initial antibiotic therapy. PPIs should be withheld 2 weeks prior to testing to avoid false negative results. ${ }^{6}$ Eradication can be confirmed using a urea breath test or a stool antigen test at least 6 weeks after completion of therapy. ${ }^{7}$ In this report, a urea breath test was used to determine the success of initial treatment; a rapid urease test was also done during follow-up endoscopy which showed a negative result. PPIs were not prescribed after $H$. $p y$ lori eradication therapy.

A histologic grading system for classifying the response of gastric MALT lymphomas to treatment was proposed by the GELA $^{5}$; this grading system classifies responses as complete histologic response, probable minimal residual disease, $r R D$, or no changes. ${ }^{5}$ In our case, histologic examination showed diffuse nodular lymphoid infiltrates and persistent focal lymphoepithelial cells, which are compatible with $\mathrm{rRD}$. In the previous study, patients classified as $\mathrm{rRD}$ were considered as non-responders. ${ }^{2}$

Eradication of $H$. pylori is the first-line therapy for gastric MALT lymphoma; however, this disease may persist in nearly $20 \%$ of patients after initial treatment thereby necessitating the use of other therapeutic approaches. Other management options include radiotherapy, chemotherapy, and watchful waiting. Radiotherapy and chemotherapy have curative potential when used for localized gastric MALT lymphoma. In patients who did not respond to initial H. pylori eradication therapy, radiotherapy and chemotherapy may be used as second-line treatments with response rates as high as $95 \%$ and $88 \%$, respectively. ${ }^{2}$ According to the guidelines, ${ }^{6,7}$ radiotherapy is the preferred option for treating localized disease. Since fractionation is important to reduce radiation toxicity, 24-30 Gy was given to the involved field over 3-4 weeks.

One report recommended a "watchful waiting" strategy for patients with residual disease as histologic regression of MALT lymphomas can be delayed for as long as 2 years after $H$. pylori eradication. ${ }^{3}$ Most patients have favorable clinical outcomes without additional treatment. ${ }^{8}$

Nakamura et al. ${ }^{2}$ reported a multicenter cohort study with long-term follow-up of 420 patients with gastric MALT lym- phoma. Among 97 non-responders (histologically rRD/no changes group) to $H$. pylori eradication therapy, 15 patients $(15 / 97,15 \%)$ were subjected to the "watchful waiting" strategy while 82 patients $(82 / 97,85 \%)$ underwent second-line treatments including radiotherapy, rituximab, and endoscopic resection. ${ }^{2}$ Most patients in the watchful waiting group failed to achieve histologic regression. ${ }^{2}$

Various factors that predict unresponsiveness to $H$. pylori eradication in gastric MALT lymphomas have been report$\mathrm{ed}^{2}$; these include a non-superficial endoscopic type of lesion, deep invasion of the gastric wall, genetic translocation $(11 ; 18) /$ API2-MALT1, proximal location, advanced stage, and absence of H. pylori. In our patient, the factors responsible for the unresponsiveness to $H$. pylori eradication may be the protruding tumor type and the tumor location in the mid-body. Unfortunately, chromosomal testing was not performed in this study; however, further molecular genetic testing may help with future diagnostic and treatment goals.

Among the various second-line treatment options for non-responders, endoscopic resection is effective and minimally invasive in skilled hands. Previous studies reported successful treatment of gastric MALT lymphomas by endoscopic mucosal resection. ${ }^{9-11}$ However, for larger lesions, ESD is superior to endoscopic mucosal resection as it allows en bloc resection of larger lesions and accurate histologic examination. To our knowledge, this is the first study that reported on the use of ESD in treating gastric MALT lymphoma. A properly selected MALT lymphoma can be cured with a single endoscopic resection thereby allowing patients to avoid concerns regarding toxicity from chemotherapy or radiotherapy. Current guidelines do not yet support endoscopic resection. With increasing evidence, endoscopic therapy can be a good alternative to chemotherapy or radiotherapy.

In conclusion, we report a case of gastric MALT lymphoma unresponsive to $H$. pylori eradication therapy that was successfully treated using ESD. Endoscopic resection may be recommended as a second-line therapy in patients with properly selected gastric MALT lymphoma as it is effective but less invasive.

Conflicts of Interest

The authors have no potential conflicts of interest.

Funding

None.

Author Contributions

Writing and approval of final manuscript: Jeongmin Choi

ORCID

Jeongmin Choi

https://orcid.org/0000-0001-6976-9887 


\section{REFERENCES}

1. Ott G, Balague-Ponz O, de Leval L, de Jong D, Hasserjian RP, Elenitoba-Johnson KS. Commentary on the WHO classification of tumors of lymphoid tissues (2008): indolent B cell lymphomas. J Hematop 2009;2:77-81.

2. Nakamura S, Sugiyama T, Matsumoto T, et al. Long-term clinical outcome of gastric MALT lymphoma after eradication of Helicobacter pylori: a multicentre cohort follow-up study of 420 patients in Japan. Gut 2012;61:507-513.

3. Wündisch T, Thiede C, Morgner A, et al. Long-term follow-up of gastric MALT lymphoma after Helicobacter pylori eradication. J Clin Oncol 2005;23:8018-8024.

4. Cheson BD, Fisher RI, Barrington SF, et al. Recommendations for initial evaluation, staging, and response assessment of Hodgkin and non-Hodgkin lymphoma: the Lugano classification. J Clin Oncol 2014;32:30593068.

5. Copie-Bergman C, Wotherspoon AC, Capella C, et al. Gela histological scoring system for post-treatment biopsies of patients with gastric MALT lymphoma is feasible and reliable in routine practice. $\mathrm{Br} J \mathrm{Hae}-$ matol 2013;160:47-52.

6. Malfertheiner P, Megraud F, O'Morain CA, et al. Management of Helicobacter pylori infection-the Maastricht V/Florence consensus report. Gut 2017;66:6-30

7. Zucca E, Arcaini L, Buske C, et al. Marginal zone lymphomas: ESMO clinical practice guidelines for diagnosis, treatment and follow-up. Ann Oncol 2020;31:17-29.

8. Ruskoné-Fourmestraux A, Fischbach W, Aleman BM, et al. EGILS consensus report. Gastric extranodal marginal zone B-cell lymphoma of MALT. Gut 2011;60:747-758.

9. Kim GH, Choi BG, Lee JN, et al. 2 cases of gastric mucosa-associated lymphoid tissue lymphoma presenting as a submucosal tumor-like lesion. Korean J Gastroenterol 2010;56:103-108.

10. Toyoda H, Ono T, Kiyose M, et al. Gastric mucosa-associated lymphoid tissue lymphoma with a focal high-grade component diagnosed by EUS and endoscopic mucosal resection for histologic evaluation. Gastrointest Endosc 2000;51:752-755.

11. Noda M, Mori N, Nomura K, et al. Regression of idiopathic thrombocytopenic purpura after endoscopic mucosal resection of gastric mucosa associated lymphoid tissue lymphoma. Gut 2004;53:1698-1700. 